\title{
Incidence of pelvic insufficiency fractures after external beam radiotherapy for gynecological cancers using post-treatment image follow-up: a meta-analysis of 3929 patients
}

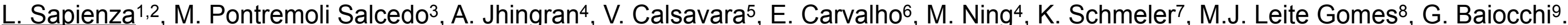 \\ ${ }_{1}^{1}$ Ascension Providence Hospital - Michigan State University College of Human Medicine, Internal Medicine, Southfield, USA \\ 2Baylor College of Medicine, Radiation Oncology, Houston, USA. \\ 3Federal University of Health Sciences/Irmandade Santa Casa de Misericordia de Porto Alegre, Obstetrics and Gynecology, Porto Alegre, Brazil. \\ 4The University of Texas MD Anderson Cancer Center, Radiation Oncology, Houston, USA. \\ ${ }^{5}$ A. C. Camargo Cancer Center, Epidemiology and Statistics, São Paulo, Brazil. \\ 6Universidade do Estado do Rio de Janeiro - UERJ, Urology, Rio de Janeiro, Brazil. \\ 7The University of Texas MD Anderson Cancer Center, Gynecologic Oncology \& Reproductive Medicine, Houston, USA. \\ 8Hospital Federal dos Servidores do Estado do Rio de Janeiro - HFSE-RJ, Radiation Oncology, Rio de Janeiro, Brazil. \\ ${ }^{9}$ A. C. Camargo Cancer Center, Gynecologic Oncology, São Paulo, Brazil.
}

Figure 1. Systematic Search

\section{Purpose:}

To estimate the overall rate, symptomatic proportion, and most common sites of pelvic insufficiency fracture (PIF) after external beam radiotherapy for gynecological cancer based on post-treatment CT, MRI, PET or bone scintigraphy.

\section{Methods:}

A systematic search of databases (PubMed and EMBASE) was performed (CRD42019125679). The pooled summary of overall PIF (detected by post treatment image follow-up) and the proportion of symptomatic cases were calculated by using the random-effects model weighted by the inverse variance.

Results:

A total of 702 articles were initially found, resulting in 21 studies that met the inclusion criteria (total 3929 patients). Five hundred and four patients presented with PIF, translating into an overall rate of $14 \%(95 \% \mathrm{Cl}: 10-18 \%$, based on 21 studies). Among these cases with PIF, the proportion of symptomatic patients was $61 \%(95 \% \mathrm{Cl}$ : $52-69 \%$, based on 14 studies). The total number of PIF was provided by 11 studies, with a total of 610 PIF (mean 1.65/ patient that develop PIF). The most common locations were: $39.7 \%$ sacro-iliac joint; $33.9 \%$ body of the sacrum; $13 \%$ pubis; $7 \%$ lumbar vertebra; $2.8 \%$ iliac bone; $2.1 \%$ acetabulum; and $1.5 \%$ femoral head/neck.

\section{Conclusions:}

The incidence of PIF after radiotherapy for gynecological cancer is high $(14 \%)$ with the majority affecting the sacral bone/joint $(73.6 \%)$. Post-treatment bone surveillance is recommended since almost forty percent of the patients were asymptomatic at the time of PIF diagnosis. Strategies to prevent the fracture in these patients are necessary.

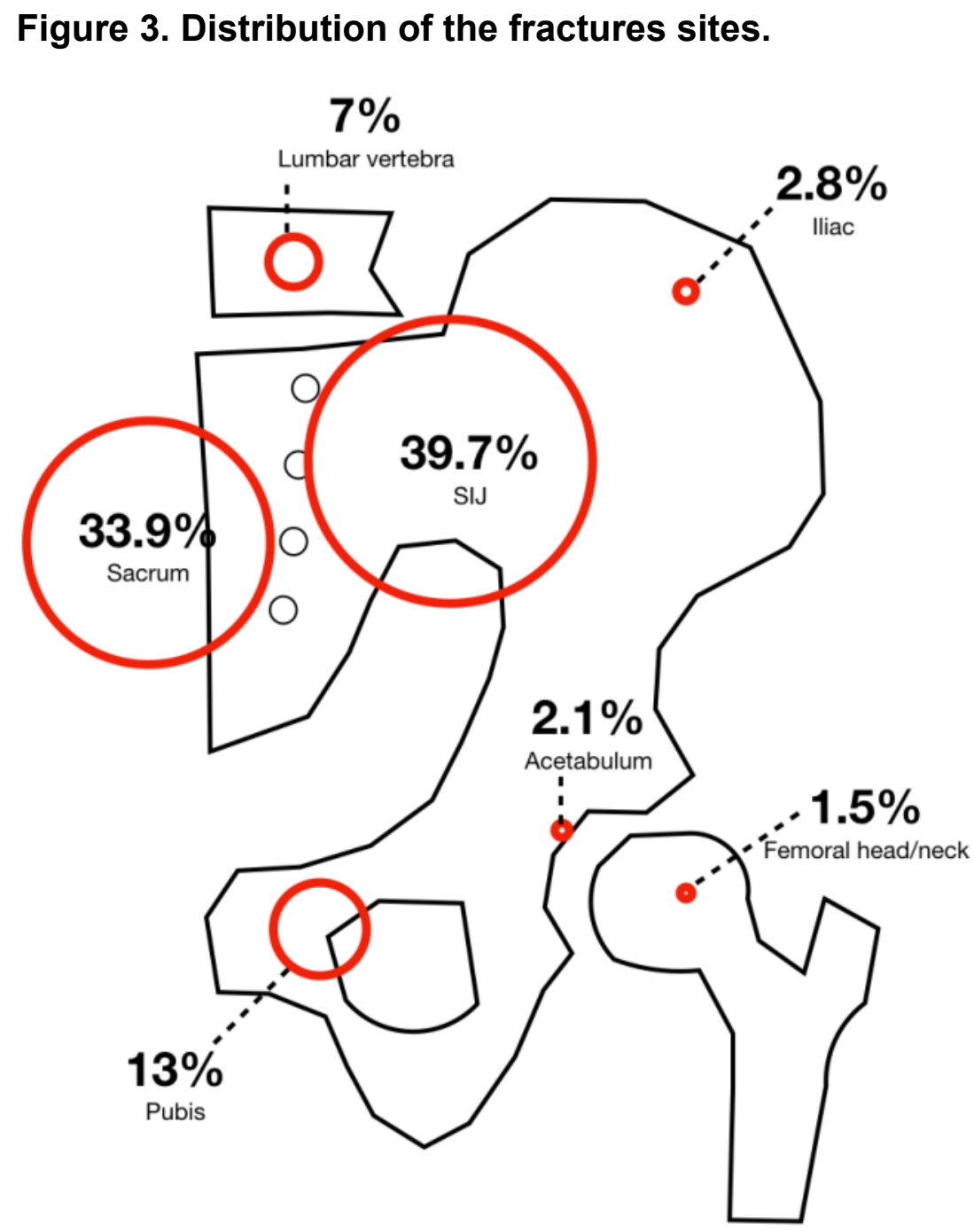

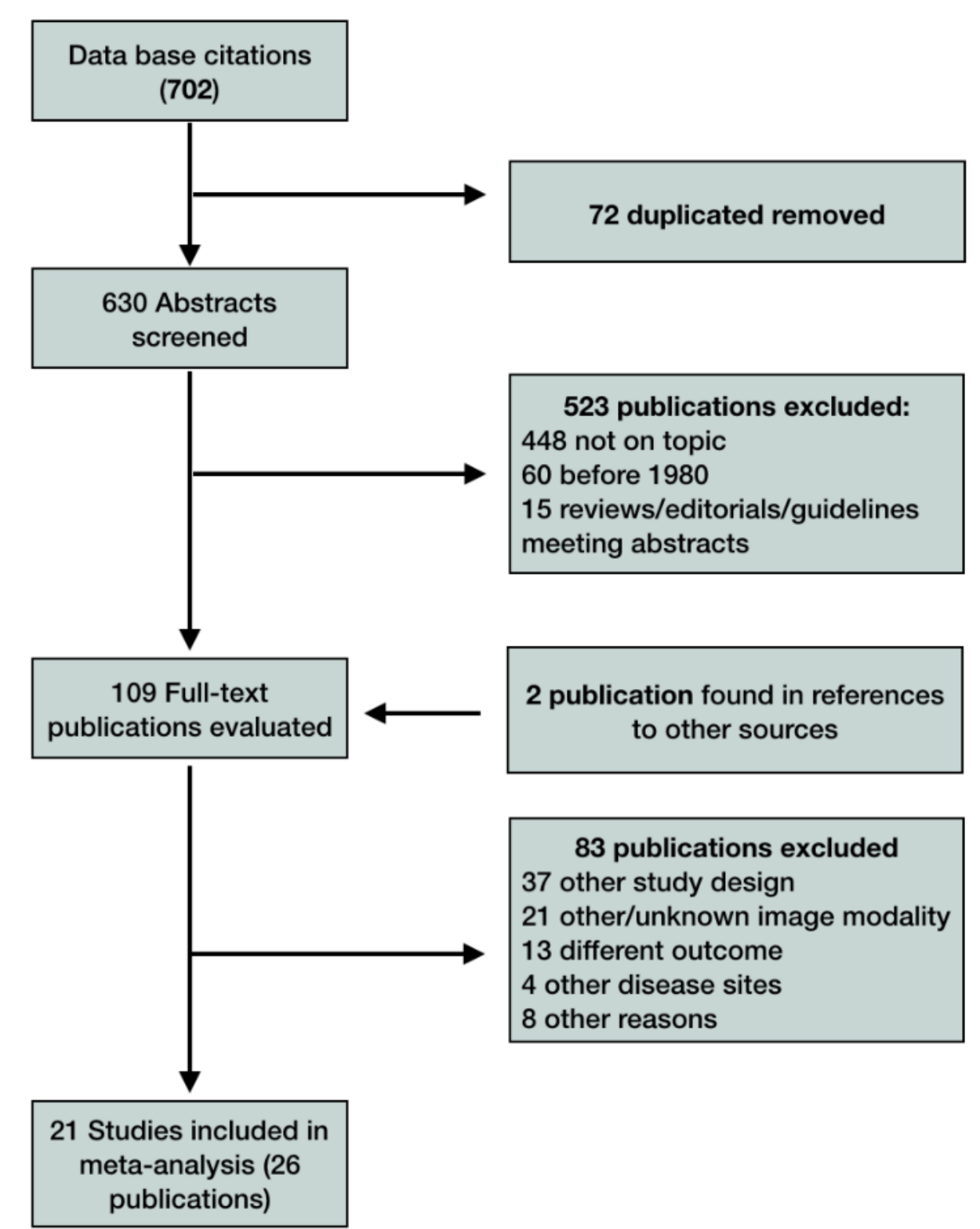

Figure 2. Meta-analysis of $(A)$ the overall rate of PIF; and $(B)$ the proportion of symptomatic cases.

A)

Study

Weidenbächer

Pontremoli Salcedo Yamamoto Ramlov Bazire

Oh 201

Kim

Mehmood

Kuji

Jung

Ueze

Shih

Park

Oh 2008

Ogino

Blomlie

Abe Random effects model

B)

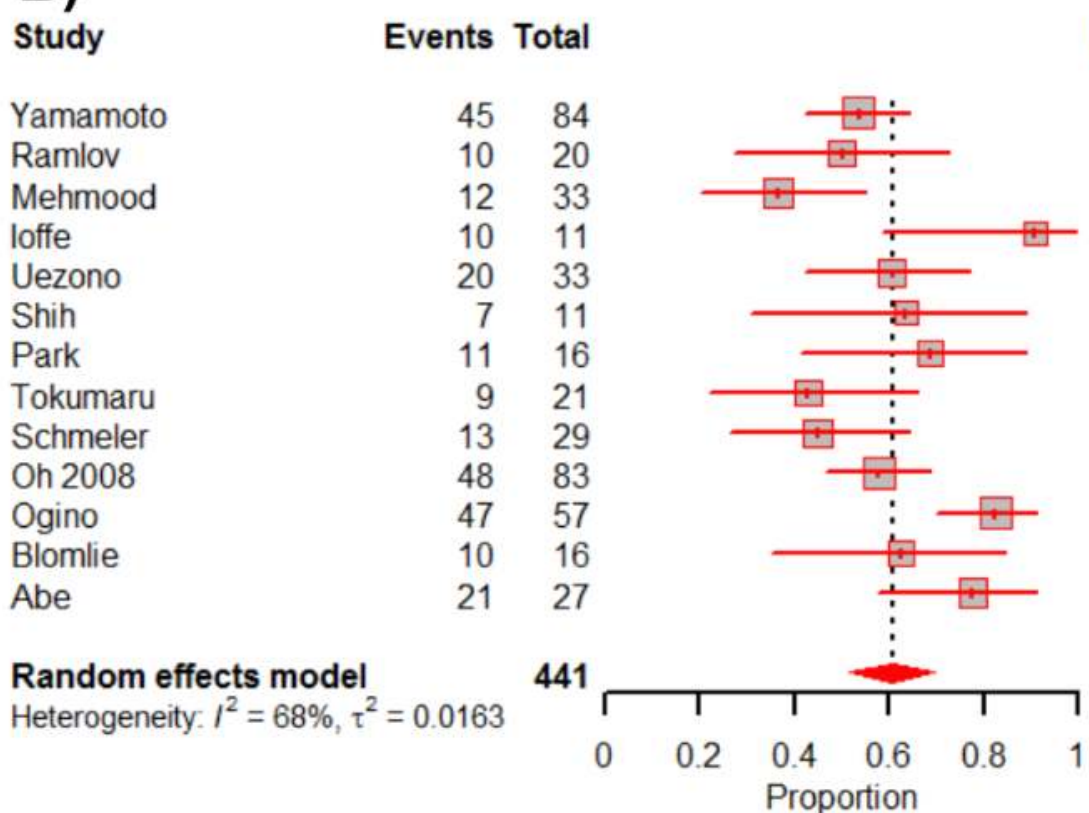

Proportion $95 \%-\mathrm{Cl}$ Weight

$0.09[0.03 ; 0.19] \quad 4.5 \%$ $0.07[0.04 ; 0.11] \quad 5.3 \%$ $0.16[0.13 ; 0.19] \quad 5.5 \%$ $0.03[0.02 ; 0.06] \quad 5.4 \%$ $0.10[0.05 ; 0.19] \quad 4.7 \%$ $0.06[0.00 ; 0.27] \quad 2.9 \%$ $0.09[0.05 ; 0.14] \quad 5.2 \%$ $0.12[0.08 ; 0.16] \quad 5.4 \%$ $0.12[0.03 ; 0.32] \quad 3.3 \%$ $07[0.04 ; 0.12] 52 \%$ $0.33[0.24,0.44] \quad 4.8 \%$ $05[0.02 ; 0.09] \quad 53 \%$ $07[0.04: 0.11] \quad 53 \%$ $0.36[0.24 ; 0.49] \quad 4.4 \%$ $0.10[0.07 ; 0.14] \quad 5.4 \%$ $0.15[0.12 ; 0.18] \quad 5.6 \%$ $0.17[0.13 ; 0.21] \quad 5.4 \%$ $0.89[0.65 ; 0.99] \quad 2.9 \%$ $0.34[0.24 ; 0.45] \quad 4.7 \%$ $0.14[0.10 ; 0.18] 100.0 \%$ 